\title{
TEOR DE SAL DOS CEREAIS DE PEQUENO-ALMOÇO E A SUA CONTRIBUIÇÃO PARA O VALOR DE REFERÊNCIA
}

\author{
SALT CONTENT IN BREAKFAST CEREALS AND ITS CONTRIBUTION TO \\ THE REFERENCE VALUE \\ CONTENIDO EN SAL DE LOS CEREALES DE DESAYUNO Y SU \\ CONTRIBUCIÓN AL VALOR DE REFERENCIA
}

\author{
Renata Souto (rsouto@ufp.edu.pt)* \\ Adriana Pimenta (apimenta@ ufp.edu.pt)** \\ Rita Catarino (ritacat@ ufp.edu.pt)*** \\ Fernanda Leal (fleal@ ufp.edu.pt)****
}

\section{RESUMO}

\begin{abstract}
As dietas contemporâneas são responsáveis por uma ingestão diária de sal muito superior ao recomendado e estão associadas a um aumento de várias patologias, das quais se destacam as doenças cardiovasculares. Neste contexto, é importante conhecer qual é a contribuição de alimentos consumidos com regularidade, como os cereais de pequeno-almoço, para o valor diário de referência (VDR) de sal. Este trabalho teve como objetivos avaliar o teor de sal nos cereais de pequeno-almoço, estimar o impacto do seu consumo no VDR e ainda avaliar a alteração do seu teor ao longo do tempo. Para isso, em 2010, foi determinado experimentalmente o teor de sal de um conjunto representativo de 13 amostras. O mesmo grupo de cereais foi monitorizado até 2019, para avaliar se existiam diferenças significativas face aos valores iniciais. Neste intervalo de tempo, verificou-se uma redução do teor de sal (valor médio de 1,1 $\pm 0,6$ para $0,8 \pm 0,4 \mathrm{~g} / 100 \mathrm{~g}$ ), apesar de se manter uma ampla variação $(0,04$ a 1,8 g/100 g). Conclui-se que uma porção de cereais, com um teor de sal médio, pode representar 3 a $7 \%$ do VDR para um adulto saudável, sendo este valor bastante superior para grupos de riscos e crianças.
\end{abstract}

Palavras Chave: cereais de pequeno-almoço, redução de sal, ingestão diária de sal, teor de sal.

\section{ABSTRACT}

Current diets are responsible for a much higher daily intake of salt than recommended and are related to an increase of several pathologies, among which are cardiovascular diseases. In this context, it is important to know the contribution of regularly consumed foods, such as breakfast cereals, to the daily reference intake (RI) of salt. The objectives of this study were to assess the salt content in breakfast cereals, to estimate the impact of their consumption on the RI and to evaluate the change in their content over time. For this, in 2010 , the salt content of a representative set of 13 samples was determined experimentally. The same group of cereals was monitored until 2019 to determine whether there were significant differences from the initial values. During this time, a reduction in the salt content (mean value $1.1 \pm 0.6$ to $0.8 \pm 0.4 \mathrm{~g} / 100 \mathrm{~g}$ ) was observed, although a wide variation was 
still maintained $(0.04$ to $1.8 \mathrm{~g} / 100 \mathrm{~g})$. Currently, a portion of cereals with a medium salt content may represent 3 to $7 \%$ of the RI for a healthy adult and a much higher value for risk groups and children.

Keywords: breakfast cereals; salt reduction, daily salt intake, salt content.

\section{RESUMEN}

Las dietas actuales son responsables de una ingestión diaria de sal muy superior a la recomendada y están asociadas a un aumento de varias patologías, de las cuales se destacan las enfermedades cardiovasculares. En este contexto, es importante conocer la contribución de alimentos consumidos con regularidad, como los cereales de desayuno, para el valor diario de referencia (VDR) de sal. Los objetivos de este estudio fueron evaluar el contenido de sal en los cereales de desayuno, estimar el impacto de su consumo en el VDR y evaluar la alteración de su contenido a lo largo del tiempo. Para esto, en 2010, se determinó experimentalmente el contenido de sal de un conjunto de 13 muestras. El mismo grupo fue monitorizado hasta 2019, para evaluar si existían diferencias significativas frente a los valores iniciales. Durante este tiempo, se verificó una reducción del contenido de sal (valor

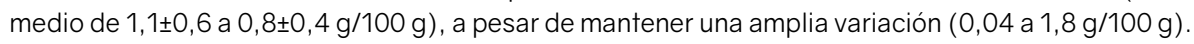
Actualmente, una porción de cereales, con un contenido de sal medio, puede representar entre el 3 y el $7 \%$ del VDR para un adulto sano, siendo este valor bastante superior para grupos de riesgo y niños.

Palabras-clave: cereales de desayuno, reducción de sal, ingesta diaria de sal; contenido de sal.

Submitted: 29th March 2019

Accepted: 08th July 2019

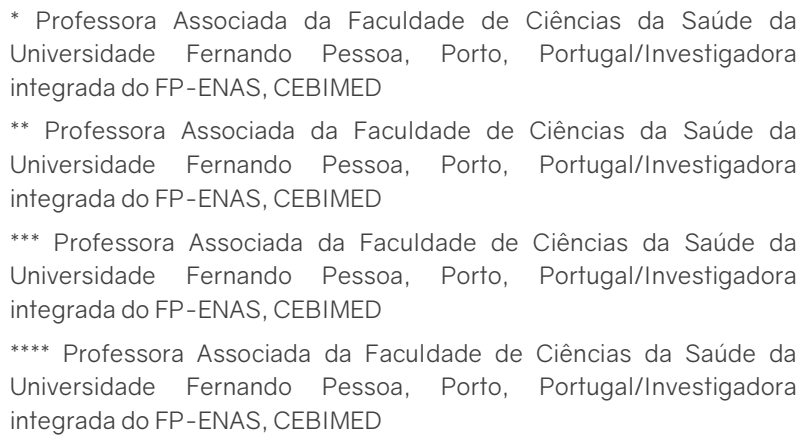




\section{INTRODUÇÃO}

O sódio $(\mathrm{Na})$ é um mineral com um papel fundamental em várias funções metabólicas. É o componente mais abundante nos fluidos extracelulares e contribui para o mecanismo de regulação da pressão arterial, balanço hídrico, contração muscular, transmissão de impulsos nervosos e absorção de nutrientes pelas células (Kloss et al., 2015; Belz et al., 2012).

A ingestão de sódio pelas sociedades ocidentalizadas excede em muito as necessidades fisiológicas e a principal contribuição vem do sal (cerca de 75-80\%), nome comumente usado para o cloreto de sódio $(\mathrm{NaCl})$. O sal está presente em todos os alimentos processados que fazem parte das dietas contemporâneas. Este composto, de baixo custo, é um dos principais contribuintes para as propriedades organoléticas dos alimentos, dado que realça o sabor de outros componentes e ajuda a minimizar o sabor amargo. Em alguns casos, tem também um papel importante em vários aspetos tecnológicos da produção de alimentos e permite estender o tempo de vida útil dos produtos (Kloss et al., 2015; Belz et al., 2012).

Os efeitos adversos associados ao elevado consumo de sal estão claramente estabelecidos. As dietas com sobrecarga de sal causam ou aumentam a pressão arterial, um importante fator de risco de doenças cardiovasculares e uma das principais causas de morbilidade e mortalidade (Public Health England, 2018). Outros problemas de saúde como albuminúria, cancro de estômago e colo-retal, asma, osteoporose e doenças renais têm, também, sido relacionados com o consumo excessivo de sal (Organização Mundial de Saúde, 2014, Kloss et al., 2015). Assim, este padrão de consumo tem elevados custos sociais e económicos para indivíduos, famílias e comunidades.

Atualmente a ingestão diária de sal é bastante superior ao valor diário de referência (VDR) indicado por organismos internacionais de saúde para uma dieta saudável: $5 \mathrm{~g}$ de sal para adultos e 3,75 g para adultos em grupos de risco (indivíduos geneticamente suscetíveis, diabéticos, hipertensos e pacientes com doença renal crónica) (Organização Mundial de Saúde, 2016; Pavlović etal., 2015; Daugirdas, 2013). Relativamente a crianças e adolescentes, estudos recentes reportam, igualmente, uma ingestão acima dos VDRs (Grimes et al., 2013; Marrero et al., 2014; Organização Mundial de Saúde, 2012; Organização Mundial de Saúde, 2013). Neste contexto, reconhece-se que uma redução da ingestão média de sal pela população (meta proposta de 30\% até 2025) traria claros benefícios para a saúde e bem-estar das populações (Organização Mundial de Saúde, 2016).

As dietas contemporâneas são amplamente baseadas em alimentos processados, sendo responsáveis por aproximadamente 75\% do consumo diário de sal (Grimes et al., 2013; Food Standard Agency, 2018). Vários estudos apontam que mais de 60\% da ingestão total de sal por dia provém de dois grandes grupos: carne/peixe processada(o) e cereais/produtos de cereais (Belz et al., 2012; Public Health England, 2018). Uma das estratégias para uma redução eficaz do consumo de sal tem sido a reformulação voluntária destes alimentos com vista a atingir uma redução gradual no seu teor de sal. Esta abordagem estimula o envolvimento da indústria alimentar e, ao mesmo tempo, permite que o consumidor se adapte a estas mudanças sem que haja uma quebra na venda dos produtos (Organização Mundial de Saúde, 2014).

Os cereais de pequeno-almoço são uma variedade de produtos derivados dos cereais, consumidos preferencialmente ao pequeno-almoço, em conjunto com outros alimentos tais como, leite, iogurtes, fruta, etc. Estes produtos, constituídos principalmente por trigo, milho e arroz, têm um paladar apelativo e são de fácil e rápida preparação. São comercializados por diferentes distribuidores/fabricantes, com uma ampla variedade de opções em relação ao processo de fabrico, formas e ingredientes adicionados. Apesar de inicialmente terem sido 
projetados para crianças, hoje em dia fazem parte da alimentação de consumidores de todas as idades (Devi et al., 2014). No geral, são excelentes fontes de energia, proteínas, vitaminas e alguns minerais (fornecem $25 \%$ da dose diária recomendada das principais vitaminas e minerais). O seu consumo regular é associado à melhoria da qualidade geral da dieta, menor índice de massa corporal e melhor desempenho mental e físico (Louie et al., 2012; Devi et al., 2014; Williams, 2014). Dado que não têm conteúdo intrínseco em sal a quantidade que apresentam é devida à adição efetuada durante a preparação da massa. O objetivo desta adição é melhorar as características organoléticas e propriedades reológicas da massa, facilitando o seu processamento (Daugirdas, 2013). Este facto justifica os resultados encontrados em vários estudos que reportam uma diferença significativa de sal nestes alimentos, existindo mesmo casos em que o mesmo produto vendido em diferentes países possui valores distintos (He etal., 2014; World Action On Salt and Health, 2016).

Por não estar localizado à superfície, o teor salino dos cereais pode ser bastante elevado, sem que, no entanto, exista um evidente sabor salgado. Neste enquadramento a redução da quantidade de sal nestes alimentos é, seguramente, conveniente e tecnicamente viável. No entanto, as características sensoriais têm de ser ajustadas para atender às expetativas dos consumidores, pois a redução de sal pode afetar tanto o sabor quanto a aparência do produto.

De acordo com a European Breakfast Cereal Association (CEEREAL), o aporte da maioria dos cereais de pequeno-almoço para o VDR de sal para um adulto é inferior a $5 \%$ (European Breakfast Cereal Association, 2016). Este valor é consistente com os 4 a $5 \%$ apontados por vários autores (Daugirdas, 2013; Grimes et al., 2013, Belz et al., 2012; Cribb et al., 2012). No caso das crianças e adolescentes, consumidores frequentes do produto, o impacto é mais significativo, uma vez que os VDR são inferiores ao dos adultos.

Tal como referido, a estratégia comum dos produtores tem sido a de efetuar mudanças lentas, não anunciadas e impercetíveis para os consumidores. Um estudo sobre as mudanças no teor de sal de alimentos processados relativo aos anos de 2003 a 2013 apontou que, dos 9 grupos alimentares principais, a maior redução percentual foi observada para os cereais de pequenoalmoço (28\%, o que equivale a uma redução de 0,31 g/100 g) (Monro et al., 2015). Os resultados do progresso da indústria alimentar na redução do teor de sal em 28 grupos de alimentos, apresentados no relatório da agência governamental Public Health England(2018), revelaram que os cereais de pequeno-almoço atingiram a meta média $(0,59 \mathrm{~g} / 100 \mathrm{~g})$ proposta pela Food Standards Agency (FSA) para 2017. Além disso, 90\% deles estavam abaixo do valor máximo (1,0 g/100 g) (Food Standards Agency, 2015). Estas metas significam uma redução de mais de $25 \%$ em relação aos valores fixados pela FSA em 2006, quando pela primeira vez publicitou metas para vários grupos de alimentos (Public Health England, 2018).

Após quase duas décadas de reduções graduais, o efeito técnico do sal remanescente chega a um nível cada vez mais crítico tornando-se, por isso, mais difícil efetuar cortes adicionais. $O$ facto de várias "receitas vencedoras" terem elevados níveis de sal e de a sua redução aumentar o custo de produção (uma diminuição de $20-30 \%$ aumenta o custo de produção em $5-30 \%$ ), pode também prejudicar os esforços de redução contínua (Kloss et al., 2015).

Deste modo, a par desta estratégia os Estados têm vindo a adotar uma série de medidas integradas e abrangentes que vão desde campanhas de sensibilização à regulamentação sobre rotulagem de géneros alimentícios (Regulamento UE № 1169/2011; Decreto-Lei n.o26/2016). Atualmente é obrigatório a indicação do teor de sal dos cereais de pequenoalmoço (em g/100 g do produto) no painel de informação nutricional (PIN), que geralmente é colocado na parte de trás ou dos lados da embalagem. Além desta informação alguns produtores/distribuidores apresentam igualmente indicações relativas à quantidade de sal em $30 \mathrm{~g}$ de produto $(\mathrm{g} / 30 \mathrm{~g})$ no PIN e/ou na frente da embalagem. Esta dose corresponde a uma 
porção média de consumo (cerca de 6 colheres de sopa) e é utilizada para estimar a contribuição dos cereais para o VDR de sal (em \%, para um adulto médio saudável). Estes sistemas de rotulagem complementares simplificados (sistemas "front-of-pack") são cada vez mais frequentes e permitem uma fácil e rápida comparação entre produtos nos locais de venda, promovendo a escolha das opções alimentares mais saudáveis.

Neste trabalho foi monitorizada a redução gradual no teor de sal de um conjunto de cereais de pequeno-almoço de consumo frequente no mercado português e avaliada a sua contribuição para os VDRs em diferentes grupos (adultos saudáveis, adultos em grupos de risco e crianças). Para isso, começou por se selecionar um conjunto representativo de amostras comercializadas e recolher os dados relativos ao seu teor de sal. Paralelamente, o conteúdo em sal destas amostras foi determinado experimentalmente e os valores obtidos foram comparados com os indicados no PIN de cada produto. Este conjunto de cereais de pequeno-almoço foi rastreado ao longo de uma década (em intervalos de 2-3 anos) para estabelecer se a reformulação destes produtos permitiu uma redução significativa do teor salino.

\section{MATERIAIS E MÉTODOS}

O presente estudo incluiu um conjunto representativo de cereais de pequeno-almoço populares entre adultos e crianças, disponível nas principais cadeias de supermercados do Porto. Estas 13 amostras foram alvo de estudo inicial em 2010 quando se recolheu informações relativas à marca, título do produto, teor de sódio/sal por $100 \mathrm{~g}$ e se determinou experimentalmente a quantidade de sal existente em cada uma.

Todas as soluções usadas no trabalho foram preparadas com reagentes de qualidade analítica e água ultrapura (condutividade específica inferior a $0,1 \mu \mathrm{S} \mathrm{cm}^{-1}$ ). A solução padrão de cloreto de sódio $\left(2,50 \mathrm{~g} \mathrm{NaCl} \mathrm{L}^{-1}\right)$ foi preparada por dissolução do sal (Merck, ref 106404100) previamente seco $\left(110^{\circ} \mathrm{C}\right.$ ). As soluções padrão de trabalho (com concentrações entre $0,125-$ $3,75 \mathrm{mg} \mathrm{NaCl} \mathrm{L}^{-1}$ ) foram preparadas diariamente por diluições rigorosas da solução padrão. A solução de ácido clorídrico concentrado (ref 84426) foi adquirida da Fluka. Esta solução foi diluída 1:1 com água ultrapura para posterior utilização na dissolução do resíduo obtido a partir da incineração da amostra.

As determinações de sódio foram realizadas num espetrofotómetro de absorção atómica Perkin Elmer (modelo Aanalyst 100), operando em modo de emissão, utilizando uma chama ar-acetileno e um comprimento de onda de 589,0 $( \pm 0,2) \mathrm{nm}$. As amostras foram incineradas de acordo com a metodologia de referência proposta pela American Association of Cereal Chemists (AACC) (AACC International, 2010) numa mufla Nabertherm (modelo 11 / B 170).

O teor de sal das amostras foi estimado por interpolação do sinal analítico obtido para as soluções de amostra preparadas a partir das cinzas na curva analítica previamente estabelecida com os padrões de trabalho. Foram efetuados triplicados para cada amostra. Os resultados obtidos neste estudo são apresentados como valor médio de $\mathrm{g}$ sal/100 g de cereal (média \pm desvio-padrão).

O mesmo grupo de cereais de pequeno-almoço foi monitorizado em intervalos regulares (de 2-3 anos). Para o tratamento e análise estatística de dados utilizou-se o software Microsoft Excel 2010 para Windows. A diferença significativa foi avaliada utilizando teste $t$ de Student, com nível de significância de 5\% (valor de $p<0,05$ ).

\section{RESULTADOS E DISCUSSÃO}


As principais características de desempenho do procedimento analítico foram primeiro avaliadas: limites de deteção e quantificação ( 51 e $127 \mu \mathrm{g} \mathrm{NaCl} \mathrm{L}^{-1}$, respetivamente), intervalo linear de trabalho $\left(0,127-5,08 \mathrm{mg} \mathrm{NaCl} \mathrm{L}^{-1}\right)$, precisão (desvios padrão relativos inferiores a $0,68 \%$ para vinte medições independentes de soluções padrão com concentrações de $\mathrm{NaCl}$ de 0,50, 1,0 e 1,5 $\mathrm{mg} \mathrm{L}^{-1}$ ) e exatidão (erros relativos compreendidos entre $-4,21 \%$ e 2,58\% para vinte medições independentes de 3 soluções padrão com concentrações de $\mathrm{NaCl}$ que abrangiam o intervalo de concentrações onde posteriormente seriam lidos os sinais referentes às soluções das amostras de cereais).

Os valores de concentração em sal obtidos experimentalmente para as amostras em estudo, bem como os declarados no PIN (em 2010), são apresentados na Tabela 1.

Tabela 1 - Teor de sal (g/100 g produto) determinado experimentalmente (concentração obtida) e indicado no painel de informação nutricional (em 2010) para o conjunto de amostras de cereais de pequeno-almoço.

\begin{tabular}{|c|c|c|}
\hline Amostra & $\begin{array}{c}\text { Concentração obtida } \\
(\mathrm{g} / 100 \mathrm{~g})^{2}\end{array}$ & $\begin{array}{c}\text { Informação nutricional (g/100 } \\
\mathrm{g})^{3}\end{array}$ \\
\hline Trigo e arroz com sabor de chocolate & $0,11 \pm 0,00$ & 0,05 \\
\hline Integrais com sabor de chocolate & $0,34 \pm 0,01$ & 0,25 \\
\hline Bolas com chocolate $^{1}$ & $0,63 \pm 0,04$ & 0,60 \\
\hline Trigo tufado com sabor de chocolate ${ }^{1}$ & $0,69 \pm 0,01$ & 0,60 \\
\hline 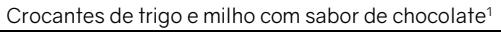 & $0,96 \pm 0,03$ & 1,0 \\
\hline Milho tufado com mel ${ }^{1}$ & $1,00 \pm 0,02$ & 1,0 \\
\hline Arroz tufado com sabor de chocolate ${ }^{1}$ & $1,06 \pm 0,04$ & 1,1 \\
\hline Integrais tostados de arroz e trigo & $1,10 \pm 0,08$ & 1,13 \\
\hline Flocos de trigo e arroz crocante com mel & $1,29 \pm 0,13$ & 1,25 \\
\hline Milho e trigo com mel & $1,30 \pm 0,05$ & 1,3 \\
\hline Trigo integral torrados com sabor a mel & $1,40 \pm 0,01$ & 1,75 \\
\hline Corn Flakes açucarados ${ }^{1}$ & $2,01 \pm 0,03$ & 1,8 \\
\hline Corn Flakes & $2,29 \pm 0,09$ & 2,38 \\
\hline
\end{tabular}

${ }^{1}$ Indicação de serem cereais infantis.

2 Os resultados são expressos como a média de três determinações independentes \pm desvio padrão.

${ }^{3}$ Informação sobre conteúdo em sal indicada no painel de informação nutricional. Quando necessário o teor equivalente em sal $(\mathrm{g} / 100 \mathrm{~g})$ foi calculado pela expressão (1):

$\mathrm{g} / 100 \mathrm{~g}=\mathrm{g}$ de sódio/100 $\mathrm{g} \times 2,5$

O teor de sal das amostras analisadas evidencia uma grande variabilidade (de 0,1 a 2,3 g/100 g), o que leva a que o contributo para o VDR dependa manifestamente do tipo de produto consumido. No caso particular das 6 amostras de cereais infantis o teor médio em sal foi de 1,1 $\mathrm{g} / 100 \mathrm{~g}$. Estes valores são consistentes com os fornecidos por outros estudos em que foram encontradas nítidas diferenças no teor de sal: 0,06 g/100 g para cereais de aveia, 0,2 g/100 g para muesli, valores de cerca de $0,8 \mathrm{~g} / 100 \mathrm{~g}$ para bolas, flocos e puffse $1 \mathrm{~g} / 100 \mathrm{~g}$ para cereais infantis (Louie etal., 2012). Outro trabalho, com 176 amostras, considera o teor médio de 0,16 $\mathrm{g} / 100 \mathrm{~g}$ para muesli, $0,79 \mathrm{~g} / 100 \mathrm{~g}$ para cereais de trigo, $0,90 \mathrm{~g} / 100 \mathrm{~g}$ para cereais infantis, 0,53 $\mathrm{g} / 100 \mathrm{~g}$ para flocos integrais, cereais com frutas, farelos e todos os outros tipos de cereais (Monro et al., 2015). Segundo estes investigadores o conteúdo médio em sal para estas amostras foi $0,54 \mathrm{~g} / 100 \mathrm{~g}$, valor ligeiramente superior ao indicado por Pavlović e colaboradores - 0,36 g/100 g - num estudo com 71 amostras de cereais de pequeno-almoço (Pavlović et al., 2015). De acordo com estes investigadores a quantidade média de sal contida em $100 \mathrm{~g}$ de amostra teria uma contribuição de 7,2\% para o VDR de um adulto saudável e 9,6\% para um grupo adulto do grupo de risco. No entanto, estas percentagens estão provavelmente sobrestimadas dado que a porção de cereais considerada é $100 \mathrm{~g}$, uma quantidade superior à que se espera ser consumida numa única ocasião. 
Para avaliar se existia uma diferença entre os valores experimentais observados e os apresentados pelos fabricantes/distribuidores no PIN efetuou-se a análise dos dados por aplicação do teste t de Student para amostras emparelhados, com um nível de significância de 0,05 (isto é, um erro de 5\%) e considerando como hipótese nula a ausência de diferença estatística entre os dois conjuntos de valores. O valor † (valor estatístico do teste) obtido é 0,0495 , menor do que o valor de $t$ crítico para o teste bicaudal que é de 2,18 , levando por isso à aceitação da hipótese nula, ou seja, não há diferença entre os dois grupos. A mesma conclusão pode ser retirada por comparação do valor de $\mathrm{p}$ (valor de prova para probabilidade bicaudal) obtido, que foi 0,961. Uma vez que p é maior que o nível de significância (ou seja, $0,05)$ aceita-se a hipótese de não haver diferença entre os valores de concentração em sal reais (obtidos através da metodologia desenvolvida) e os apresentados no PIN. Este facto legitima os procedimentos utilizados pelos fabricantes para a obtenção do conteúdo em sal e possibilita o acompanhamento das alterações salinas destas mesmas amostras ao longo do tempo por comparação direta dos valores constantes no painel nutricional.

Para que os compromissos da indústria alimentar sobre reduções graduais em sal dos alimentos processados possam ocorrer é necessário considerar espaços de tempo suficientemente alargados. O objetivo principal é adotar uma abordagem gradual de diminuição do teor de sódio, com vista a adaptar/reeducar as preferências de paladar do consumidor ao longo do tempo, sem uma quebra no consumo do produto. A prática comum é, por isso, monitorar o conteúdo em intervalos de tempo regulares, suficientemente espaçados (mínimo de cerca 2 anos), para que as alterações possam ser efetivadas. Neste trabalho, o mesmo conjunto de cereais foi reexaminado em 2013, 2015, 2017 e 2019 (Tabela 2).

Tabela 2 - Teor de sal (g/100 g produto) indicado no painel de informação nutricional em diferentes anos para 0 mesmo conjunto de cereais de pequeno-almoço

\begin{tabular}{|c|c|c|c|c|c|}
\hline \multirow[b]{2}{*}{ Amostra } & \multicolumn{5}{|c|}{ Informação nutricional $(\mathrm{g} / 100 \mathrm{~g})^{2}$} \\
\hline & 2010 & 2013 & 2015 & 2017 & 2019 \\
\hline Trigo e arroz com sabor de chocolate & 0,05 & 0,05 & 0,05 & 0,06 & 0,04 \\
\hline Integrais com sabor de chocolate & 0,25 & 0,38 & 0,38 & 0,38 & 0,32 \\
\hline Bolas com chocolate $^{1}$ & 0,60 & 0,60 & 0,80 & 0,50 & 0,50 \\
\hline Trigo tufado com sabor de chocolate ${ }^{1}$ & 0,60 & 0,5 & 0,5 & 0,45 & 0,45 \\
\hline Crocantes de trigo e milho com sabor de chocolate $^{1}$ & 1,0 & 1,0 & 0,80 & 0,84 & 0,78 \\
\hline Milho tufado com mel ${ }^{1}$ & 1,0 & 0,75 & 0,5 & 0,40 & 0,75 \\
\hline Arroz tufado com sabor de chocolate ${ }^{1}$ & 1,1 & 1,1 & 0,76 & 0,75 & 0,90 \\
\hline Integrais tostados de arroz e trigo & 1,13 & 1,00 & 1,00 & 1,00 & 1,00 \\
\hline Flocos de trigo e arroz crocante com mel & 1,25 & 1,25 & 1,03 & 1,11 & 1,03 \\
\hline Milho e trigo com mel & 1,3 & 1,3 & 1,3 & 1,25 & 1,25 \\
\hline Trigo integral torrados com sabor a mel & 1,75 & 1,35 & 1,05 & 1,07 & 0,98 \\
\hline Corn Flakes açucarados ${ }^{1}$ & 1,8 & 1,8 & 1,8 & 0,75 & 0,75 \\
\hline Corn Flakes & 2,38 & 2,30 & 2,30 & 1,80 & 1,80 \\
\hline
\end{tabular}

Indicação de serem cereais infantis.

${ }^{2}$ Informação sobre conteúdo em sal indicada no painel de informação nutricional. Quando necessário o teor equivalente em sal foi calculado pela expressão (1).

Observa-se que, durante este espaço de tempo, a reformulação dos produtos permitiu uma restrição da amplitude de concentração em sal entre as amostras (de 0,05 a 2,38 g/100 g em 2010 para 0,04 a 1,80 g/100 g em 2019) que representa uma retração em cerca de $25 \%$ do intervalo inicialmente detetado.

Das 13 amostras seguidas, 12 registaram uma redução média da quantidade de sal entre 2010 e 2019 de 22\% (mínimo de 3,8\% e máximo de 58\%), havendo apenas uma amostra em que se 
detetou um aumento de $28 \%$ relativamente ao valor de 2010 (correspondente a um aumento de $0,07 \mathrm{~g} / 100 \mathrm{~g}$ ). O perfil de variação para esta amostra (cereais integrais com sabor de chocolate) leva a supor que após um período inicial de redução em sal (para um valor de 0,25 g/100 g em 2010), terá havido uma quebra na aceitação do produto por parte do consumidor, razão pela qual em anos subsequentes houve um ligeiro aumento (para 0,38 g/100 g) tendose agora iniciado uma nova fase de diminuição progressiva (valor atual de 0,32 g/100 g). Outras amostras evidenciaram também ligeiras oscilações no padrão de decréscimo continuado ao longo de quase uma década (Tabela 2), possivelmente pela razão já apontada, apesar de uma redução no teor de sal relativamente a 2010 ter sido atingida (Figura 1). A amplitude desta redução levou a decréscimos entre 0,01 e 1,05 g/100 g de produto (que correspondem a reduções percentuais no produto de 20,0 e $58,3 \%$, respetivamente).

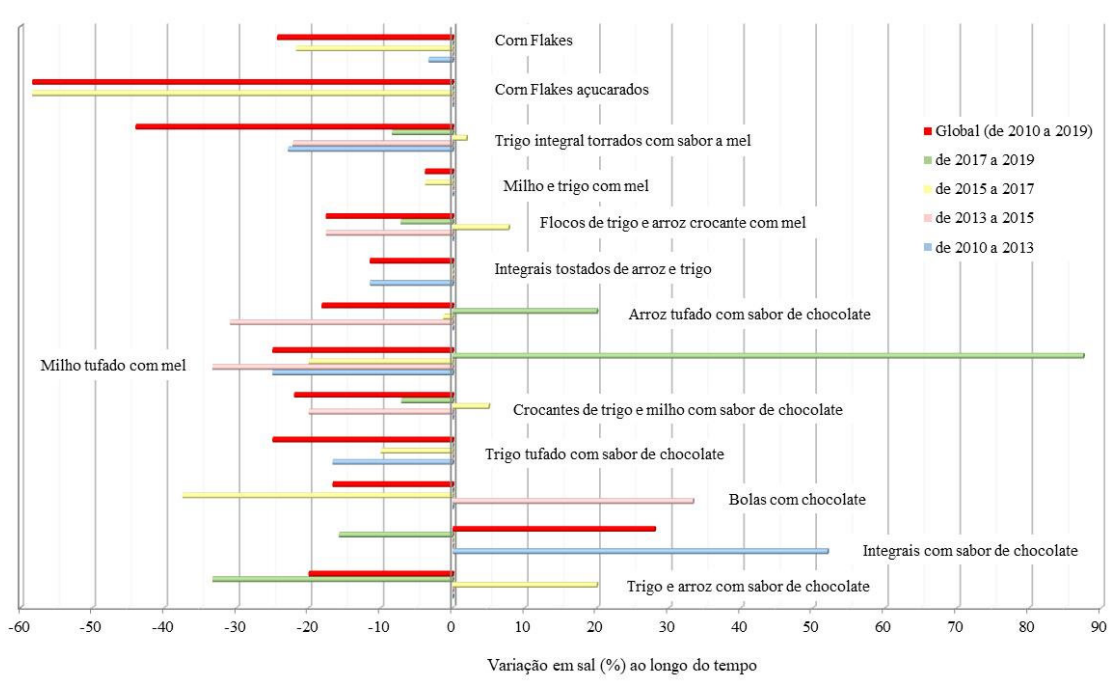

Figura 1 - Variação percentual do teor de sal do conjunto de amostras de cereais de pequeno-almoço em estudo ao longo dos anos em relação ao valor apresentado em 2010.

Para verificar se houve uma redução estatisticamente relevante neste intervalo de tempo, considerou-se as quantidades em sal em 2019 e 2010 e repetiu-se o teste t de Student para amostras emparelhadas, para um nível de significância de 0,05, sendo a hipótese nula não haver alteração do teor médio em sal. Existem diferenças significativas já que o valor de † $(3,14)$ é maior que o valor de $\dagger$ crítico para teste bicaudal $(2,18)$ e o valor de $p(0,0043)$ menor que 0,05 .

Efetuando a mesma comparação entre ciclos de análise consecutivos (isto é, 2010-2013, 2013-2015, 2015-2017 e 2017-2019) não é possível rejeitar a hipótese nula (valores de † sempre inferiores a valor de † crítico e valores de $p>0,05$ ), ou seja, não se pode considerar ter havido alteração no conteúdo em sal nestes curtos intervalos de tempo. Apesar disso, comparando a média do teor de sal e o respetivo desvio padrão para o conjunto de amostras em cada um destes ciclos detetou-se sempre uma redução com uma concomitante diminuição da amplitude das concentrações: 1,09 $\pm 0,64,1,03 \pm 0,60(3), 0,94 \pm 0,59(9), 0,80 \pm 0,46$ e 0,81 $\pm 0,44 \mathrm{~g} / 100 \mathrm{~g}$ para os anos de 2010, 2013, 2015, 2017 e 2019, respetivamente. Estes resultados são consistentes com o facto, já referido, de as reduções efetuadas pela indústria serem muito graduais para que sejam impercetíveis para o consumidor. 
Do mesmo modo, o teste t de Student aplicado a análise de dados referente aos anos de 2010 e 2015 também não permitiu a rejeição da hipótese nula e apenas quando se alarga o intervalo para 7 anos (ou seja, considerando os valores de 2010 e 2017) é possível detetar diferenças significativas (valor de $t=3,17$ e valor de $p$ bicaudal $=0,008$ ). Este facto, confirma a necessidade de monitorar os alimentos processados durante espaços de tempo suficientemente alargados, para que as ajustes no teor de sal efetuados pelos fabricantes nos seus produtos possam ser considerados significativos.

Para avaliar a contribuição atual de cada amostra estudada para o VDR em sal considerou-se o consumo de uma porção diária de $30 \mathrm{~g}$ e 5 grupos populacionais distintos: um adulto saudável, um adulto pertencente a um grupo de risco ou uma criança de 3 grupos etários diferentes. A Organização Mundial de Saúde recomenda que a ingestão máxima de referência para crianças entre os 2 e os 15 anos seja ajustada para valores inferiores à dos adultos (< de $5 \mathrm{~g} / \mathrm{dia}$ ) com base nas suas necessidades energéticas, mas não aponta valores de referência. Marrero e colaboradores num estudo transversal com crianças, propuseram VDRs para crianças em faixas etárias distintas tendo por base o valor indicado pelo Comité Científico Consultivo sobre Nutrição do Reino Unido (Scientific Advisory Committee on Nutrition, SACN) para adultos e efetuando ajustes a esse valor de acordo com a área média da superfície corporal das crianças (Marrero et al., 2014). O consumo de referência diário para as crianças proposto foi: $2 \mathrm{~g}$ para crianças de 3 a 4 anos, $3 \mathrm{~g}$ para a faixa de 5 a 8 anos, $4 \mathrm{~g}$ para crianças dos 9 aos 11 anos e após essa idade (adolescentes) idêntico ao dos adultos. $O$ valor de referência de $3,75 \mathrm{~g} /$ dia foi considerado para os adultos em grupos de risco (Pavlović et al., 2015; Daugirdas, 2013).

O aporte de sal devido ao consumo de uma dose de $30 \mathrm{~g}$ de amostra para o VDR de cada grupo considerado foi então estimado (Tabela 3). Estes valores representam, por isso, o papel individual do sal existente nessa porção para dose de sal diária referenciada. 
Tabela 3 - Contribuição expressa em percentagem do consumo de uma porção de $30 \mathrm{~g}$ de cereal de pequenoalmoço para o valor diário de referência (VDR) do sal.

\begin{tabular}{|c|c|c|c|c|c|c|}
\hline & & & & \\
\hline & & & & \multicolumn{3}{|c|}{$\begin{array}{l}\text { Contribuição (em \%) para o } \\
\text { valor diário de referência do } \\
\text { sal }^{3}\end{array}$} \\
\hline Amostra & $\begin{array}{c}\mathrm{g} / 30 \mathrm{~g} \\
\text { de } \\
\text { produt } \\
\mathrm{O}^{2}\end{array}$ & $\begin{array}{c}\text { adulto } \\
\text { saudáv } \\
\text { el } \\
\text { (5 g } \\
\text { /dia) }\end{array}$ & $\begin{array}{c}\begin{array}{c}\text { adulto } \\
\text { de }\end{array} \\
\text { grupo } \\
\text { de } \\
\text { risco } \\
(3,75 \\
\text { g/dia) }\end{array}$ & $\begin{array}{c}\text { criança } \\
\text { de } 3-4 \\
\text { anos } \\
(2 \\
\text { g/dia) }\end{array}$ & $\begin{array}{c}\text { criança } \\
\text { de } 5-8 \\
\text { anos } \\
\text { (3 } \\
\text { g/dia) }\end{array}$ & $\begin{array}{c}\text { criança } \\
\text { de } 9- \\
11 \text { anos } \\
\text { (4 } \\
\text { g/dia) }\end{array}$ \\
\hline Trigo e arroz com sabor de chocolate & 0,012 & 0,24 & 0,32 & 0,60 & 0,40 & 0,30 \\
\hline Integrais com sabor de chocolate & 0,096 & 1,92 & 2,56 & 4,80 & 3,20 & 2,40 \\
\hline Bolas com chocolate $^{1}$ & 0,150 & 3,00 & 4,00 & 7,50 & 5,00 & 3,75 \\
\hline Trigo tufado com sabor de chocolate ${ }^{1}$ & 0,135 & 2,70 & 3,60 & 6,75 & 4,50 & 3,38 \\
\hline 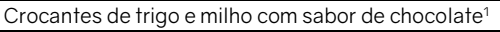 & 0,234 & 4,68 & 6,24 & 11,70 & 7,80 & 5,85 \\
\hline Milho tufado com mel ${ }^{1}$ & 0,225 & 4,50 & 6,00 & 11,25 & 7,50 & 5,63 \\
\hline Arroz tufado com sabor de chocolate ${ }^{1}$ & 0,270 & 5,40 & 7,20 & 13,50 & 9,00 & 6,75 \\
\hline Integrais tostados de arroz e trigo & 0,300 & 6,00 & 8,00 & 15,00 & 10,00 & 7,50 \\
\hline Flocos de trigo e arroz crocante com mel & 0,309 & 6,18 & 8,24 & 15,45 & 10,30 & 7,73 \\
\hline Milho e trigo com mel & 0,375 & 7,50 & 10,0 & 18,75 & 12,50 & 9,38 \\
\hline Trigo integral torrados com sabor a mel & 0,294 & 5,88 & 7,84 & 14,70 & 9,80 & 7,35 \\
\hline Corn Flakes açucarados $^{1}$ & 0,225 & 4,50 & 6,00 & 11,25 & 7,50 & 5,63 \\
\hline Corn Flakes & 0,540 & 10,80 & 14,40 & 27,00 & 18,00 & 13,50 \\
\hline
\end{tabular}

Indicação de serem cereais infantis.

${ }^{2}$ Informação (de 2019) sobre sal indicada no painel de informação nutricional e/ou no painel simplificado na frente na embalagem. Quando necessário o teor de sal por $30 \mathrm{~g}(\mathrm{~g} / 30 \mathrm{~g})$ de produto foi calculado pela expressão (2):

$$
\mathrm{g} / 30 \mathrm{~g}=\mathrm{g} / 100 \mathrm{~g} \times 0,30
$$

${ }^{3}$ Os valores indicados entre parênteses correspondem ao valor diário de referência (VDR) de sal considerado para cada grupo. A contribuição (em \%) foi calculada pela expressão (3):

$\%$ VDR $=\frac{\mathrm{gde} \mathrm{sal} / 30 \mathrm{~g} \text { de produto }}{\text { VDR }} \times 100$

De acordo com a classificação do teor de sal dos alimentos proposto pela FSA, apenas 2 das amostras avaliadas (em 2019) podem ser consideradas como tendo um baixo teor de sal ( $\leq 0,3$ $\mathrm{g}$ sal/100 g), a maioria é considerada de teor médio (> 0,3 g/100 g a $\leq 1,5 \mathrm{~g} / 100 \mathrm{~g}$ ) e um produto tem alto teor de sal (>1,5 g/100 g) (Food Standard Agency, 2016).

Se apenas forem considerados os cereais de teor médio em sal para o cálculo do VDR, verificase que o consumo de uma porção pode representar 3 a 7\% deste valor para um adulto saudável.

Por outro lado, apesar das reduções alcançadas com a reformulação dos produtos, continuam a existir discrepâncias acentuadas no conteúdo em sal das diferentes amostras, o que leva a que o consumo de uma porção de $30 \mathrm{~g}$ do produto em estudo que detinha o maior conteúdo em sal (Corn Flakes) represente um aporte de 10,8\% para o mesmo grupo (isto é, para um adulto saudável).

Os resultados alcançados permitem ainda inferir que, seja qual for a situação, a contribuição do sal pode, de facto, ser bastante significativa dado que o tipo de amostra tem uma notória interferência. São ainda relatados teores de sal díspares para o mesmo produto. He et al. (2014) reportaram que no Canadá o All-Bran da Kellogg's possuía um teor de sal de 2,15 g/100 g enquanto que nos Estados Unidos o valor era de apenas 0,65 g/100 g. A mesma informação foi veiculada numa pesquisa internacional que corrobora a enorme variação no teor de sal do mesmo produto vendido em diferentes países revelando que, por exemplo, os Cornflakes da Kellogg's vendidos na Índia continham o mais elevado teor de sal (1,93 g/100 g), sendo este um valor $46 \%$ superior ao do mesmo produto comercializado na Argentina e no Brasil $(1,04$ g/100 g) (World Action On Salt and Health, 2016). 
O contributo dos cereais de pequeno-almoço para o VDR em sal é ainda fortemente dependente da faixa etária em causa. Considerando apenas o conjunto de cereais publicitado como infantis, verifica-se que para crianças de 3-4 anos a sua contribuição mínima é de 7,50\% (valor médio para as 6 amostras de 10,3 $\pm 2,6 \mathrm{~g} / \mathrm{dia}$ ), uma quantidade que não pode ser desprezada. Para o grupo de crianças de 5-8 anos e 9-11 anos, a \% média para o VDRs destas amostras é $6,88 \pm 1,75 \mathrm{~g} /$ dia e 5,16 $\pm 1,31 \mathrm{~g} /$ dia, respetivamente, valores que também não podem ser tidos como insignificantes. Este contributo elevado para a \%VDR foi também evidenciado num trabalho com 340 crianças dos 5 a 17 anos (Marrero et al., 2014). Este estudo reportou que aproximadamente $70 \%$ dos participantes ingeria uma quantidade diária de sal superior ao VDR e que os cereais de pequeno-almoço representavam $6 \%$ desse total. Um outro estudo, com 4487 crianças e adolescentes dos 2-16 anos apontou que, independentemente do nível socioeconómico e da idade, o consumo de sal era maior que o desejado (6,1 a 6,6 g/dia) e que os cereais de pequeno-almoço representavam 4,2\% do VDR (Grimes et al., 2013).

\section{CONCLUSÃO}

Os cereais de pequeno-almoço têm presentemente um papel relevante na alimentação de crianças e adultos. Estes alimentos são excelentes fontes de energia, proteínas, vitaminas e alguns minerais, estando o seu consumo regular associado à melhoria da qualidade geral da dieta, menor índice de massa corporal e melhor desempenho mental e físico.

Apesar dos benefícios apresentados, estes produtos podem contribuir com uma \% significativa para o VDR de sal. No caso dos adultos, o consumo de uma porção de um cereal classificado com teor médio (o que representa a maioria das amostras de cereais) pode facilmente atingir 3 a $7 \%$ do VDR. No entanto, este valor fica subestimado quando a porção é superior a $30 \mathrm{~g}$, a frequência de consumo é superior a uma vez por dia ou, ainda, se o produto consumido tiver um teor de sal superior ao valor médio. Em relação às crianças, o contributo de uma porção (30 g) é ainda mais significativo já que os valores das \%VDRs, comparativamente com os adultos, são duas vezes superiores. Assim, a contribuição em sal dos cereais, dependendo da situação, pode ser significativa e, portanto, não deve ser negligenciada.

Em conclusão, os consumidores de cereais de pequeno-almoço devem estar cientes da grande diversidade do teor em sal dos produtos existentes no mercado. A rotulagem melhorada, com sinalização nutricional clara e abrangente na frente da embalagem dos alimentos tem aqui um papel fundamental. Atualmente, a maior parte dos cereais de pequenoalmoço inclui um rótulo nutricional voluntário simplificado, em que as informações sobre a quantidade de sal por porção de produto são também expressas em \%VDR para um adulto médio saudável. Este esquema complementar é uma plataforma importante para a comunicação com o consumidor, pois possibilita comparações fáceis e rápidas entre os produtos no momento da compra, incentivando a escolha informada de alternativas mais equilibradas e saudáveis.

A redução substancial no teor de sal dos cereais de pequeno-almoço ao longo do tempo é inegável. Este progresso deve-se às iniciativas implementadas pelos fabricantes, consideradas um exemplo de sucesso. No entanto, são necessários esforços adicionais em todo o setor alimentar para que a meta da OMS de redução da ingestão média de sal em $30 \%$ até 2025 seja atingida. Para o cumprimento deste objetivo, além da redução do teor de sal nos alimentos, é importante a adoção de mais políticas integradas e abrangentes, como são exemplos as iniciativas de conscientização da população para a adoção de uma alimentação e 
estilo de vida saudáveis e a melhoria, desenvolvimento e implementação de legislação alimentar relativa à rotulagem e às alegações nutricionais e de saúde sobre os alimentos.

\section{BIBLIOGRAFIA}

AACC International. (2010). Approved Methods of Analysis (11th ed.). St. Paul, Minnesota: American Association of Cereal Chemists International.

Belz, M., Ryan, L. \& Arendt, E. (2012). The impact of salt reduction in bread: a review. Critical Reviews in Food Science and Nutrition, 52(6), 514-524. doi: 10.1080/10408398.2010.502265

European Breakfast Cereal Association. (2016). Breakfast Cereals - A great way to start the day. Disponível em: http://www.ceereal.eu/why-breakfast/breakfast-cereals

Cribb, V., Warren, J. \& Emmett, P. (2012). Contribution of inappropriate complementary foods to the salt intake of 8-month-old infants. European Journal of Clinical Nutrition, 66(1), 104-110. doi: 10.1038/ejcn.2011.137

Daugirdas, J. (2013). Potential Importance of Low-Sodium Bread and Breakfast Cereal to a Reduced Sodium Diet. Journal of Renal Nutrition, 23(1), 1-3. doi: 10.1053/j.jrn.2012.03.002.

Decreto-lei no 26/2016 de 9 de junho. Diário da República no 111/2016 - I Série. Lisboa: Ministério da Agricultura; Florestas e Desenvolvimento Rural.

Devi, A., Eyles, H., Rayner, M., Ni Mhurchu, C., Swinburn, B., Lonsdale-Cooper, E. \& Vandevijvere, S. (2014). Nutritional quality, labelling and promotion of breakfast cereals on the New Zealand market. Appetite, 81(1), 253-260. doi: 10.1016/j.appet.2014.06.019

Food Standards Agency. (2015). Salt reduction targets for 2017. Disponível em: http://www.foodstandards.gov.scot/2017-salt-targets

Food Standards Agency. (2016). Guide to creating a front of pack (FoP) nutrition label for prepacked products sold through retail outlets. Disponivel em: https://www.food.gov.uk/sites/default/files/media/document/fop-guidance_0.pdf

Food Standards Agency. (2018). Salt. Disponível em: https://www.food.gov.uk/businessguidance/salt

Grimes, C., Campbell, K., Riddell, L. \& Nowson, C. (2013). Is socioeconomic status associated with dietary sodium intake in Australian children? A cross-sectional study. BMJ Open, 3(2), 1-7. doi: 10.1136/bmjopen-2012-002106

He, F., Brinsden, H. \& MacGregor, G. (2014). Salt reduction in the United Kingdom: a successful experiment in public health. Journal of Human Hypertension, 28(6), 345-352. doi: 10.1038/jhh.2013.105

Kloss, L., Meyer, J., Graeve, L. \& Vetter, W. (2015). Sodium intake and its reduction by food reformulation in the European Union - A review. NFS Journal, 1, 9-19. doi: 10.1016/j.nfs.2015.03.001

Louie, J., Dunford, E., Walker, K. \& Gill, T. (2012). Nutritional quality of Australian Breakfast cereals. Are they improving?. Appetite, 59(2), 464-470. doi: 10.1016/j.appet.2012.06.010

Marrero, N. M., He, F. J., Whincup, P. \& MacGregor, G. A. (2014). Salt Intake of Children and Adolescents in South London: Consumption Levels and Dietary Sources. Hypertension, 63(5), 911912. doi: 10.1161/hypertensionaha.113.02264

Monro, D., Mhurchu, C., Jiang, Y., Gorton, D. \& Eyles, H. (2015). Changes in the Sodium Content of New Zealand Processed Foods: 2003-2013. Nutrients, 7(6), 4054-4067. doi: 10.3390/nu7064054

Organização Mundial De Sáude. (2012). WHO Guideline: Sodium intake for adults and children. Disponível em:

https://www.who.int/nutrition/publications/guidelines/sodium_intake_printversion.pdf 
Organização Mundial De Sáude. (2013). Mapping salt reduction initiatives in the WHO European Region. Disponivel em: http://www.euro.who.int/__data/assets/pdf_file/0009/186462/Mappingsalt-reduction-initiatives-in-the-WHO-European-Region.pdf

Organização Mundial de Saúde. (2014). European Food and Nutrition Action Plan 2015-2020. Disponível

em: http://www.euro.who.int/_data/assets/pdf_file/0008/253727/64wd14e_FoodNutAP_140426.pdf Organização Mundial de Saúde. (2016). Salt reduction. Disponível em: https://www.who.int/news$\mathrm{room} /$ fact-sheets/detail/salt-reduction

Pavlović, L., Popović, M., Bijelović, S., Velicki R. \& Torović, L. (2015). Salt Content in Ready-to-Eat Food and Bottled Spring and Mineral Water Retailed in Novi Sad. Srpski Arhiv Za Celokupno Lekarstvo (Serbian Archives of Medicine), 143(5-6), 362-368. doi: 10.2298/SARH1506362T

Public Health England. (2018). Salt targets 2017: progress report. Disponível em: https://www.gov.uk/government/publications/salt-targets-2017-progress-report.

Regulamento (UE) n' 1169/2011 do Parlamento Europeu e do Conselho, de 25 de outubro de 2011 relativo à prestação de informação aos consumidores sobre os géneros alimentícios. Jornal oficial da união europeia L 304/18-63.

Williams, P. (2014). The Benefits of Breakfast Cereal Consumption: A Systematic Review of the Evidence Base. Advances in Nutrition, 5(5), 636S-673S. doi: 10.3945/an.114.006247

World Action on Salt and Health. (2016). New International Survey Reveals Huge Differences in the Sugar and Salt Content of Global Breakfast Cereal Brands. Disponível em: http://www.worldactiononsalt.com/news/surveys/2016/ 
